\title{
Integration of 4G LTE and DSRC (IEEE 802.11p) for Enhancing Vehicular Network Performance in IoV Using Optimal Cluster-Based Data Forwarding (OCDF) Protocol
}

\author{
https://doi.org/10.3991/ijim.v15i14.19201 \\ Shaik Mazhar Hussain $(\bowtie)$ \\ Universiti Teknologi Malaysia (UTM), Johor Bahru, Malaysia, \\ Middle East College, Muscat, Oman \\ hussain@graduate.utm.my \\ Kamaludin Mohamad Yusof, Shaik Ashfaq Hussain \\ Universiti Teknologi Malaysia (UTM), Malaysia, Johor Bahru \\ Rolito Asuncion \\ Middle East College, Muscat, Oman \\ Syed Ghouse \\ Jawaharlal Nehru Technological University (JNTU), Hyderabad, India
}

\begin{abstract}
IoV}$ is a known platform for exchanging data between vehicles and distinct networks through diverse communication media. Embedded technologies like IoT and Intelligent Transportation, are aimed to build smart networks for IoV to support diverse automated applications such as smart vehicle control, intelligent traffic control, and dynamic data services. However, in the smart domain, the implementation of IoV has unresolved challenges. The synchronization of vehicles and humans is a crucial issue in making decisions. Therefore, a proper understanding of the pertinent issues about IoV implementation that can improve the VNs performance is essential. DSRC and cellular networks are considered as potential alternatives for endorsing V2X communications. DSRC is employed for intelligent and automotive transportation applications through short-range data exchange between DSRC-components. Although spectrum assigned to DSRC alone will not be appropriate to satisfy huge information traffic needs for internet access in vehicles. Cellular networks offer potential solutions, attributing an extensive range of cell coverage, broadly deployed infrastructure, and greater capacity. Nevertheless, the centralized characteristics of these networks limit the ability to handle low-latency communications that can challenge the efficacy of several safety applications. This paper reviews potential DSRC and wireless integrated solutions for efficient vehicular communications. In methodology, first, we reviewed existing technologies that integrate DSRC with other wireless technologies, and secondly, the study is carried out to highlight the limitations for each supporting vehicular communications. Thereby, the paper embeds a brief comparative analysis. Finally, an algorithm is proposed to integrate DSRC and 4G-LTE with a novel Optimal Cluster-
\end{abstract}


ing-based Data Forwarding Protocol for efficient data transmission to attain better performance in IoV. The performance of the proposed algorithm is compared with the existing approaches using the NS-3 simulation tool. The obtained results demonstrate that the proposed algorithm outperforms the existing approaches in terms of End-to-End Delay and throughput.

Keywords-Internet of Vehicles (IoV); 4G/LTE; DSRC; IoT; Vehicular Networks

\section{Introduction}

The Internet of Vehicles is a network of vehicles used that are used to exchange information via various radio access technologies. Information exchange can happen between vehicle to vehicle, vehicles to road side unit, vehicle to pedestrian, vehicle to infrastructure, vehicle to everything $(\mathrm{X})$. IoV is considered as a full stack of VANET's. One of the major concerns associated with the Intelligent Transportation System (ITS) is the increasing traffic congestions, especially in urban scenarios which seriously impacts safety, energy consumption, air pollution, noise disturbance, and travelling time [8], [10], [14]. Hence, it is necessary to optimize, control, and monitor vehicular flows to benefit the users around the globe. Continuous advancements in the transportation industry has led to the development of connected vehicles which forms the key enablers to the ITS. Hence, the concept of VANET's has transformed into a smart concept of IoV. IoV has been developed to enable various applications in addition to the current vehicle sensing abilities for road safety and vehicle traffic control. Some of the applications include collision warning, collision avoidance, and vehicle diagnostics. The V2X communications had enhanced the collaboration among pedestrians, infrastructures, and vehicles thereby promising to reduce $85 \%$ of road accidents thereby fostering automobile sectors for intelligent and safer transportation systems. In IoV, vehicles gather data from the surroundings facilitating other vehicles for pollution control, traffic control, and safe navigation. However, communication can be upgraded by advanced wireless communication technologies compatible with conventional and forthcoming applications of VANET. Among the available wireless communication technologies, DSRC, and cellular networks are considered as the potential technologies because of their enormous benefits. DSRC offers low latency suitable for safety applications but has link degradation problems due to obstacles and also from channel collisions because of high vehicle density. In contrast, LTE networks has wide coverage range and supports bandwidth applications requiring higher data rates and reliability. However, it has high delays which leads to high transmission time intervals and may not be suitable for safety applications. To support wide range of road transport applications and to ensure safety road environments, integrating DSRC and LTE is necessary to enhance the V2X services for future ITS. In our work, we have proposed integrated solution using two algorithms for data dissemination and congestion control. Moreover, to evaluate the performance, three use cases have been considered - safety, bandwidth and VoIP services. In [1], [7], [12] a hetero- 
geneous solution is proposed for IoV using different use cases, however, failed to evaluate the packet delivery ratio and latency. In [2] [3], Authors have proposed hybrid architectures where DSRC and cellular networks are used for vehicular communications. Hybrid approach is proposed in [4] focusing on video streaming applications based on the RAT selection. However, the performance is evaluated considering only two vehicles with no competition on bandwidth. Hence, it is required to investigate more real time scenarios considering vehicles transmission under high vehicle densities. Aforementioned approaches usually selects the radio access technologies by switching assuming LTE has unlimited resources. In the coming future, V2X applications may face variety of service requirements. Safety messages must be transmitted frequently with low latencies unlike bandwidth greedy services. However, bandwidth greedy services may not be active all the time unless triggered by certain events. Hence, Single technology would support for different service requirements. Hence, it is essential to make the RAT policy service oriented. The research work proposes cluster enabled data forwarding protocol for efficient data dissemination with radio interface selection algorithm enabling heterogeneous solution which selects radio technology according to the service requirements with the consideration of network performance.

The main contribution of this research work are

1. Proposing an optimized cluster-based data forwarding protocol for efficient data dissemination.

2. Intelligently monitoring and assessing packet loss ratio levels using radio interface selection algorithm

3. Superiority of the proposed algorithm will be evaluated and compared with existing approaches using NS-3

The proposed work is implemented using C-ITS standards and validated using NS3 simulation tool. Three use cases were considered - safety services, bandwidth services, and voice services under urban environment considering packet delivery ratio (PDR), delay, throughput, and latency as the major performance metrics. The findings of my research work shows the superiority of the proposed approach and outperforms the existing approaches and, also the transmission of safety messages are supported with low latency.

The remaining part of this paper is organized as follows:

Section 2 discusses about the existing works, and then a brief comparison is articulated. Section 3 addresses the research question and Section 4 discusses the proposed approach. Section 5 discusses about the results and followed by discussions in section 6. Section 7 concludes the paper and highlights the future work. Finally, references are given in Section 8. 


\section{$2 \quad$ Literature Review}

A comprehensive review of the existing works is thoroughly studied and detailed conclusions were drawn. Table 1 shows the summary of the existing works highlighting the findings and remarks.

Table 1. Summary of Existing Works

\begin{tabular}{|c|c|c|}
\hline Paper & Findings & Remarks \\
\hline 1 [3] & $\begin{array}{l}\text { WiMAX-DSRC is integrated. The clustering technique } \\
\text { is applied to enhance the throughput. The performance } \\
\text { metric used for evaluation is throughput. }\end{array}$ & $\begin{array}{l}\text { The need for improving the cluster head } \\
\text { selection technique for attaining better } \\
\text { results in further work was highlighted. }\end{array}$ \\
\hline $2[4]$ & $\begin{array}{l}\text { 4G-LTE and DSRC are integrated. Three applications } \\
\text { are considered: collision avoidance, message broadcast, } \\
\text { and multimedia file download. From the results, It is } \\
\text { observed that 4G/LTE is preferred for non-safety } \\
\text { applications whereas DSRC is preferred for safety } \\
\text { applications. Under collision avoidance scenario, } \\
\text { DSRC outperforms 4G/LTE. For non-safety message } \\
\text { broadcast, both are acceptable. For multimedia file } \\
\text { download, LTE outperforms DSRC. }\end{array}$ & $\begin{array}{l}\text { The experiments are conducted with few } \\
\text { vehicles. The experiments were done indi- } \\
\text { vidually for DSRC and 4G/LTE. It is rec- } \\
\text { ommended to integrate DSRC and 4G LTE } \\
\text { for connected vehicles to achieve better } \\
\text { performance. }\end{array}$ \\
\hline $3[5]$ & $\begin{array}{l}\text { A novel low cost sensor based system is developed for } \\
\text { road safety applications in Intelligent Transportation } \\
\text { System (ITS). An embedded wireless sensor network } \\
\text { based embedded system is developed. In this work, } \\
\text { three scenarios were considered - speed bump detec- } \\
\text { tion, detecting sharp turns and detection of rain and } \\
\text { fog. }\end{array}$ & $\begin{array}{l}\text { Only two vehicles were considered for the } \\
\text { V2V platform. The reduction in packet size } \\
\text { would improve transmission speed, packet } \\
\text { losses, and network congestion. Appropri- } \\
\text { ate compression algorithms could substan- } \\
\text { tially reduce packet size. }\end{array}$ \\
\hline 4 [6] & $\begin{array}{l}\text { ZigBee, Wi-Fi, and DSRC communication technolo- } \\
\text { gies performance was investigated for deployment in } \\
\text { the IoV system. It is found that all three technologies } \\
\text { offer successful data exchange with a failure rate of } 1 \% \\
\text { for low-frequency communications. Whereas, At } \\
\text { higher frequencies, Wi-Fi and DSRC offer this perfor- } \\
\text { mance. It is observed from the simulation results, Data } \\
\text { exchange rate of Wi-Fi is good as it has the highest } \\
\text { data rates and channel bandwidth as compare to DSRC } \\
\text { and ZigBee. ZigBee does not support packet size of } \\
\text { greater than } 300 \text { Bytes as the transmission delay will } \\
\text { exceed } 100 \mathrm{~ms} \text {. Hence, QoS requirements are not met } \\
\text { due to packet loss of more than } 1 \% \text {. }\end{array}$ & $\begin{array}{l}\text { The frequency of messages and maximum } \\
\text { packet size depends on the mechanism of } \\
\text { application. In ITS, It is very important to } \\
\text { adapt effective IoV solutions for road safety } \\
\text { and traffic management as they are depend- } \\
\text { ent on the reliability and performance of the } \\
\text { V2V and V2I communication channels. } \\
\text { Hence, Selecting appropriate wireless } \\
\text { communication technology is critical for } \\
\text { implementing a feasible IoV system de- } \\
\text { signed for road safety and traffic manage- } \\
\text { ment applications. }\end{array}$ \\
\hline $5[13$ & $\begin{array}{l}\text { Link aware High Data Transmission (LHT) approach } \\
\text { has been proposed for high data transmission applica- } \\
\text { tions. Signal strength and bandwidth are considered as } \\
\text { the evaluation metrics for the selection of next nodes } \\
\text { and data about vehicle nodes. The proposed approach } \\
\text { is performed with existing approaches and accordingly, } \\
\text { simulations are performed. From the results, It is } \\
\text { shown that the proposed approach has outperformed } \\
\text { the existing approaches. The LHT is proposed for } \\
\text { video transmission and high data rate applications. }\end{array}$ & $\begin{array}{l}\text { The approach is suggested for high data } \\
\text { transmission applications such as video } \\
\text { transmission applications and not for safe- } \\
\text { ty-related applications where QoS plays a } \\
\text { vital role. Only a few performance metrics } \\
\text { were evaluated and hence there is a need to } \\
\text { test with more performance metrics. The no } \\
\text { of vehicle nodes considered is very less. } \\
\text { Though the application belongs to high data } \\
\text { rates and the impact of considering more } \\
\text { vehicles in congested areas for high data } \\
\text { transmission applications is not discussed. }\end{array}$ \\
\hline
\end{tabular}




\begin{tabular}{|c|c|c|}
\hline 6 [9] & $\begin{array}{l}\text { Heterogeneous IoV architecture for V2I is proposed } \\
\text { where multiple interfaces were incorporated such as } \\
\text { Wireless Access in Vehicular Environments (WAVE), } \\
\text { Wi-Fi, and 4G/LTE. Best Interface Algorithm (BIA) is } \\
\text { proposed to select the best wireless interface for relia- } \\
\text { ble communication to support seamless connectivity } \\
\text { required for efficient data forwarding in V2I communi- } \\
\text { cation. }\end{array}$ & $\begin{array}{l}\text { Two performance metrics were evaluated: } \\
\text { Throughput and Latency by varying simula- } \\
\text { tion time and mobility speed. It is observed } \\
\text { that heterogeneous architecture outper- } \\
\text { formed individual wireless interfaces and } \\
\text { also conventional WAVE architectures. The } \\
\text { major concern in this paper is the perfor- } \\
\text { mance of the algorithm is evaluated for } 30 \\
\text { vehicles. Hence, the impact of traffic con- } \\
\text { gestion on such heterogeneous architectures } \\
\text { is still challenging. This could urge the need } \\
\text { of developing more efficient, reliable, and } \\
\text { smart algorithms that can minimize access } \\
\text { control issues. }\end{array}$ \\
\hline $7[11]$ & $\begin{array}{l}\text { Multitier heterogeneous adaptive vehicular (MHAV) } \\
\text { networks are proposed. In this paper, network scaling } \\
\text { and load balancing between DSRC and LTE are inves- } \\
\text { tigated. From the results obtained, It is shown that end } \\
\text { to end communication delay to be less than } 50 \mathrm{~ms} \text { is } \\
\text { above } 90 \% \text { for a density of } 250 \text { vehicles } / \mathrm{k}\end{array}$ & $\begin{array}{l}\text { There is still a challenge that persists in } \\
\text { investigating highway scenarios with } \\
\text { MHAV. }\end{array}$ \\
\hline $8[13]$ & $\begin{array}{l}\text { Vehicular Floating Car Data Collection (FCD) is } \\
\text { proposed for collecting massive amounts of data. LTE } \\
\text { and DSRC were integrated called a hybrid approach } \\
\text { offering superior performance. In this cellular radio, } \\
\text { access will be offloaded. A lightweight signaling } \\
\text { procedure is developed based on DSRC VANET to } \\
\text { avoid duplicate data records and hence reducing } \\
\text { VANET load. Hence, avoids interfering with other } \\
\text { VANET-specific messages. }\end{array}$ & $\begin{array}{l}\text { One major limitation of this paper is due to } \\
\text { offloading LTE access network, there is an } \\
\text { increase in data transferring delay. The } \\
\text { challenge is how to cope with this increase } \\
\text { in data transferring delay under high vehicle } \\
\text { density scenarios? Considering the same } \\
\text { hybrid approach. }\end{array}$ \\
\hline \multirow[t]{2}{*}{9 [15] } & $\begin{array}{l}\text { The paper has proposed hybrid architecture called the } \\
\text { Vehicular Multihop algorithm for Stable Clustering } \\
\text { (VMaSC-LTE) integrating DSRC based multihop } \\
\text { clustering and the Long-Term Evolution (LTE) to } \\
\text { attain a high data packet delivery ratio (PDR) and low } \\
\text { delay while keeping the cellular architecture usage at a } \\
\text { minimum level. Cluster Head (CH) selection is based } \\
\text { on the average relative speed concerning the neighbor- } \\
\text { ing vehicles. The performance metrics considered in } \\
\text { this paper are Data Packet Delivery Ratio, delay, } \\
\text { control overhead, and clustering stability. In this paper, } \\
\text { IEEE 802.11p-LTE hybrid architecture is proposed } \\
\text { where vehicles form multihop clustered topology in } \\
\text { each direction of the road. The average relative speed is } \\
\text { considered a clustering metric. The paper does not } \\
\text { investigate the use of the proposed algorithm in urban } \\
\text { scenarios. }\end{array}$ & $\begin{array}{l}\text { The proposed algorithm is not investigated } \\
\text { for urban traffic scenarios where the major } \\
\text { challenge would be traffic congestions. }\end{array}$ \\
\hline & & $\begin{array}{l}\text { Performance metrics for evaluation are } \\
\text { throughput, transmission delay, dissemina- } \\
\text { tion delay, Packet Delivery Ratio. Perfor- } \\
\text { mance of integrated DSRC and 4G/LTE } \\
\text { will be compared with DSRC alone and } \\
\text { 4G/LTE alone. The experiments would } \\
\text { probably be carried out under sparse and } \\
\text { high-density networks to evaluate the } \\
\text { performance of the proposed algorithm. }\end{array}$ \\
\hline
\end{tabular}




\section{Research Question}

1. How does the performance of vehicular networks will affect when no of vehicles in certain area increases?

2. Will the DSRC networks alone be able to fulfill the latency requirements under high vehicle density scenarios for safety applications?

3. Does the performance of DSRC remain the same when no of vehicles increases within its coverage area?

\section{Proposed Approach}

The major issues concerning DSRC are low latency, and link degradation. Some of the limitations in VANET architecture with respect to data forwarding applications are connectivity, switching, and availability of bandwidth. To address these issues, heterogeneous IoV architectures are proposed in the existing works to achieve better results and to mitigate the challenges pertaining to network unavailability, increased user demands, network connections, and higher bandwidth. However, due to lower data rates, low latency has made such networks ineffective and failed to attain the outcomes. Hence, the performance of real-time traffic environments is reduced enormously. Also, most of the previous works have used only single technology which has degraded the overall network performance, for instance, DSRC alone. Thus, it is necessary to integrate multiple technologies to enhance vehicular network performance and achieving better results. In this work, we have integrated DSRC and 4G LTE to handle intelligent transportation systems and enhancing the communication infrastructures. We have proposed optimal cluster-based data forwarding protocol for efficient message dissemination in vehicle to infrastructure in heterogeneous IoV. In our work, first we introduce an improved beetle swarm optimization algorithm for the selection of optimal cluster heads and clustering that improves the quality of data transfer in terms of energy efficiency, data loss, and delay. The cluster member forwards the data to the cluster head and then forwards to the corresponding radio access unit. Then, we proposed radio interface selection algorithm to illustrate congestion control technique at service layer. Finally, three types of use-cases are considered- Safety services, Bandwidth greedy services, and VoIP services.

1. Network Layer - Optimal cluster-based data forwarding protocol for efficient data transferring

2. Service Layer - Congestion control through Efficient Radio Interface Selection algorithm

3. Application Layer - Safety, bandwidth services, and VoIP services 

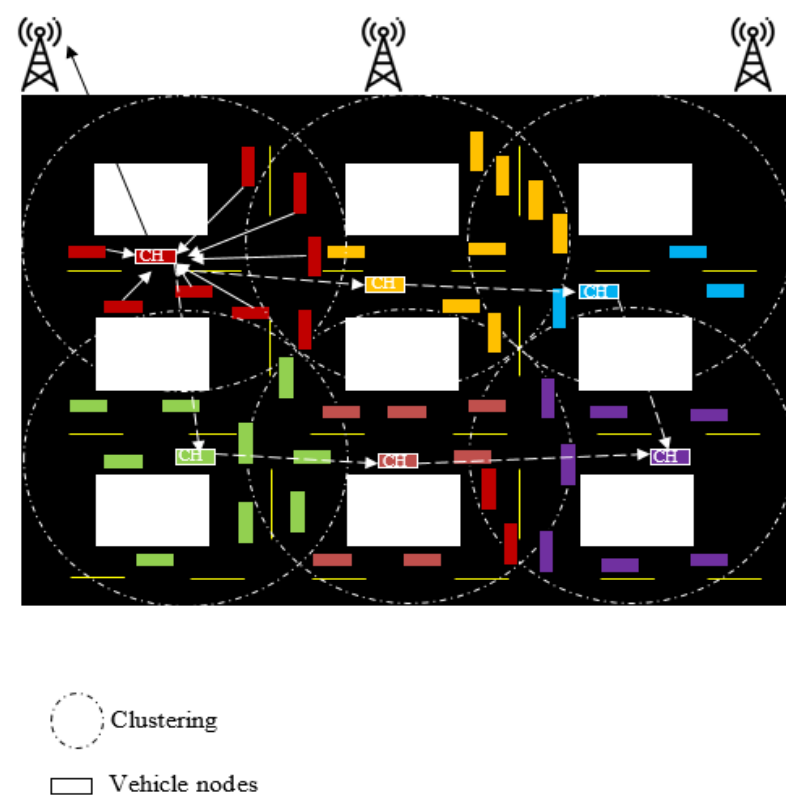

Fig. 1. Network model of proposed OCDF protocol

Algorithm I: Optimized Cluster Based Data Forwarding (OCDF) protocol for efficient data transfer

Energy aware clusters are created using an improved beetle swarm optimization algorithm for optimal selection of cluster heads. The main purpose is to resolve the issues related to the packet delivery rate, end-to-end delay as well as latency. The algorithm is explained in four different steps:

1. Initialization Phase

2. Fitness calculation

3. Updating solution

4. Termination Phase

Besides these, there are two important phases which are explained as follows:

Neighbor discovery phase: Each nodes sends a welcome packet that contains its identification and the vehicular nodes updates the neighboring table with the identity quality signal value in the welcome packets received from the other vehicles.

Flooding phase: In this phase, every node has its identification, energy, just as neighboring broadcasting. As the data received, cluster heads are selected based on their residual energy, where the node with the energy over the average network potential will be selected as cluster head, and hence selecting the ideal $\mathrm{k} \mathrm{CHs.} \mathrm{The} \mathrm{average}$ power of all VNs is given as:

$$
E N_{A}=\frac{\sum_{i}^{X} E N_{i}}{X}
$$


Anywhere, ' $\mathrm{X}$ ' is the number of active $\mathrm{VNs},{ }^{\prime} E N_{i}{ }^{\prime}$ 'residual energy for ' $V N_{i}{ }^{\prime}$, after that control station use IBSO algorithm to discover optimal k CHs. The subsequent processing steps are utilized to develop an optimal solution.

1. Initialization: Optimal CHs are selected using the improved beetle swarm optimization algorithm. Optimal $\mathrm{CHs}$ are selected as follows. The $\mathrm{CHs}$ are represented as $\mathrm{CH}_{i}=\left\{\mathrm{CH}_{1}, \mathrm{CH}_{2}, \mathrm{CH}_{3}, \mathrm{CH}_{4}, \mathrm{CH}_{5}\right\}$. Each node represents the solution or optimal multipath routes in the network. Here, the VNS are represented as,

$$
Z_{i}=\left(Z_{i}^{1}, \ldots \ldots Z_{i}^{d}, \ldots \ldots \ldots Z_{i}^{n}\right)
$$

Solution creation means that initiation is an important step in the optimization process, which enables you to quickly recognize the optimal solution. The obtained solution is given for the following stage i.e., fitness evaluation.

2. Fitness calculation: Once the solution is generated, the fitness function is evaluated and then chooses the best solution. Selection of the fitness is a fundamental aspect in IBSO.

Where,

$$
\text { Fitness }_{i}=\min \{\text { Distance, Energy }
$$

Signifies the fitness value. Here, the fitness value is calculated utilizing the parameters average distance as well as residual energy. A representative through minimum fitness value has heavier mass and has better position, i.e., the better is the cluster head selection. The obtained results are given for the next step i.e. updating solution.

3. BSO based updating solution: In this part, each beetle determines a potential solution to the optimization problem. Similar to the particle swarm algorithm, the beetles also share the information, but the distance as well as direction of the beetles are determined by their speed and the intensity of the information to be detected by their long antennae. In mathematical form, we took the idea of particle swarm algorithm. There is a population of $\mathrm{n}$ beetles represented as $P=$ $\left(P_{1}, P_{2}, \ldots . P_{n}\right)$ in an $\mathrm{S}$-dimensional search space, where $\mathrm{i}^{\text {th }}$ beetle represents an $\mathrm{S}$ dimensional vector $P_{i}=\left(P_{i 1}, P_{i 2}, \ldots . P_{i s}\right)^{r}$, represents the position of the $\mathrm{i}^{\text {th }}$ beetle in $Q_{i}=\left(Q_{i 1}, Q_{i 2}, \ldots . . Q_{i s}\right)^{r}$ the $\mathrm{S}$-dimensional search space and also represents a potential solution to the problem. According to the target function, the fitness value of each beetle position can be calculated. The speed of the $i^{\text {th }}$ beetle is expressed as. The individual extremity of the beetle is represented as $U_{i}=$ $\left(U_{i 1}, U_{i 2}, \ldots . U_{i s}\right)^{r}$ and the group extreme value of the population is represented as $U_{g}=\left(U_{g 1}, U_{g 2}, \ldots \ldots . U_{g s}\right)^{r}$. The mathematical model for simulating its behavior is as follows;

$$
P_{i s}^{k+1}=P_{i s}^{k}+\lambda \xi_{i s}^{k}+(1-\lambda) \xi_{i s}^{k}
$$


Where

$\mathrm{K} \rightarrow$ specify the current number of iterations,

$Q_{i s} \rightarrow$ expressed as the speed of beetles,

$\varepsilon_{i s} \rightarrow$ represents the increase in beetle position movement. Here, the speed formula is mathematically represented as follows;

$$
Q_{i s}^{k+!}=\omega Q_{i s}^{k}+C_{1} r_{1}\left(U_{i s}^{k}-P_{i s}^{k}\right)+c_{2} r_{2}\left(U_{g s}^{k}-P_{g s}^{k}\right)
$$

Where,

$C_{1}, C_{2} \rightarrow$ two optimistic constants,

$r_{1}, r_{2} \rightarrow$ represents the arbitrary functions inside the range $[0,1]$,

$\omega \rightarrow$ represents the inertia weight.

This paper adopts the strategy of decreasing inertia weight, and which is mathematically represented as follows;

$$
\omega=\frac{\omega_{\max }-\omega_{\min }}{K} * k
$$

Where,

$\omega_{\min }, \omega_{\max } \rightarrow$ denotes the minimum as well as maximum value of $\omega$,

$\mathrm{k}, \mathrm{K} \rightarrow$ denotes the present number of iterations as well as the maximum number of iterations,

4. Termination phase: The algorithm terminates if an ideal determination of $\mathrm{CH}$ is accomplished as well as the arrangement which is holding the best value is picked and it is resolved as the best solution. Weight of the cluster heads are determined based on the following condition,

$$
W\left(V N_{j}, C H_{i}\right)=a \frac{E_{r e s}\left(C H_{i}\right)}{d\left(V_{j}, C H_{i}\right) \times d\left(C H_{i}, B S\right)}
$$

Where, $E_{\text {res }}\left(\mathrm{CH}_{i}\right)$ represents the residual energy of $\mathrm{CH}$. VNs link to the $\mathrm{CH}$ through advanced residual energy.

$\frac{1}{d\left(V_{j}, C H_{i}\right)}$, represents mutual of distance among VN in addition to $\mathrm{CH}$. The VN joins to the adjacent $\mathrm{CH}$ in its communication range.

$\frac{1}{d\left(\mathrm{CH}_{i}, \mathrm{BS}\right)}$ - defines the reciprocal of distance among $\mathrm{CH}$ as well as base station. The $\mathrm{VN}$ is link to the $\mathrm{CH}$, which is closer to the base station BS.

'a' represents a stable value.

During this formation of clusters, every VN calculates this weight esteem utilizing the above condition. At that point the $\mathrm{VN}$ joins to the $\mathrm{CH}$ with the most noteworthy weight value.

Algorithm II: Congestion Control through Efficient Radio Interface Selection algorithm at Service layer.

In our proposed work, it is assumed that the vehicles are interfaced with two radio access technologies DSRC, and LTE for transmitting and receiving information. The network selection is based on the network performance, and QoS requirements. Three application scenarios were considered- safety, non-safety, and bandwidth greedy ap- 
plications. The dedicated interfaces guarantees to provide low latency safety service and high bandwidth services. Hence, to achieve this, appropriate selection of network interfaces are necessary. DSRC signals are used for the transmission of safety messages, and LTE is used for high bandwidth services. The network performance is assessed by continuously monitoring Packet Delivery Ratio (PDR) levels. The reason of considering PDR for assessing the network performance is due to the link degradation problem, and channel collusion in DSRC resulting in reduced PDR at higher loads. Hence, to address this, PDR threshold values are set for DSRC-LTE that is PDRThDSRC and PDRThLTE. In case of other service messages, PDR levels will be checked by the proposed protocol. Assuming that LTE service is charged, primarily DSRC will be chosen for Other Service. It will be switched to LTE only if $<<$ PDRLTE $\leq$ PDRThLTE $>>$ and $<<$ PDRDSRC $>$ PDRThDSRC $>>$ indicating overloaded DSRC but not LTE.

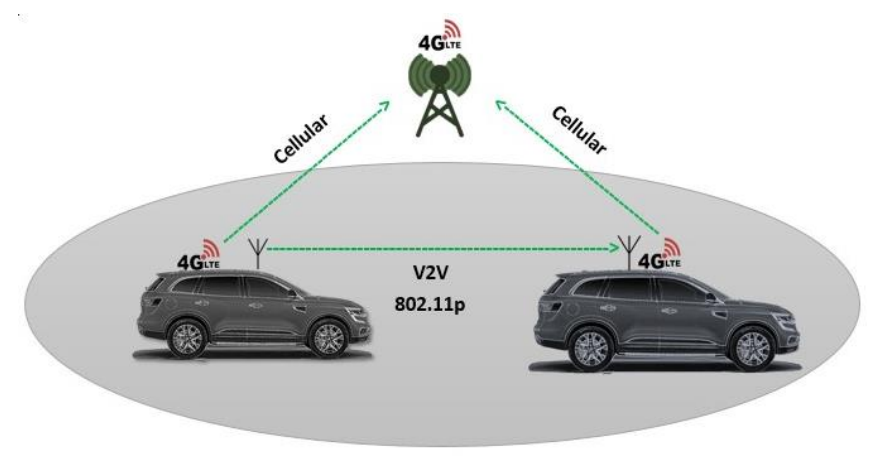

Fig. 2. Integration of DSRC and LTE for V2V communication

\section{$5 \quad$ Simulation Results}

The performance of the proposed research work is simulated using NS-3 software simulation tool. Throughput and delay are considered as the performance metrics to evaluate the superiority of the proposed algorithm. It is assumed in our work that the vehicles are equipped with two terminals 4 G LTE and DSRC. Both the terminals have the ability to transmit and receive the information. The proposed algorithm is compared with the existing 4G LTE, WAVE, LR-Wifi, and heterogeneous solution. In our work, we have assumed three application scenarios- voice, video and safety messages with a packet size of 500B and video size of up to $1 \mathrm{~KB}$ and a vehicle density of 600 nodes. Figure 3 and 4 shows the delay and throughput performance of existing and proposed approach. 


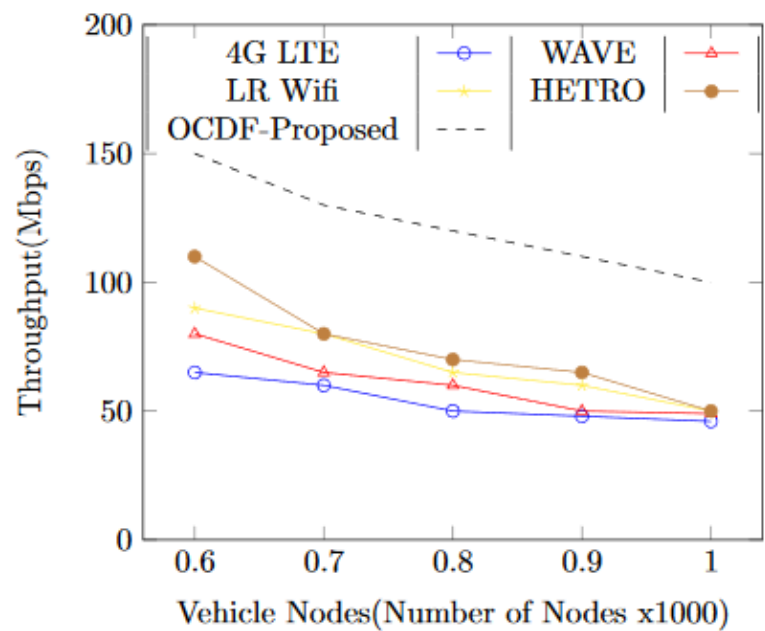

Fig. 3. Vehicle nodes vs Throughput [9]

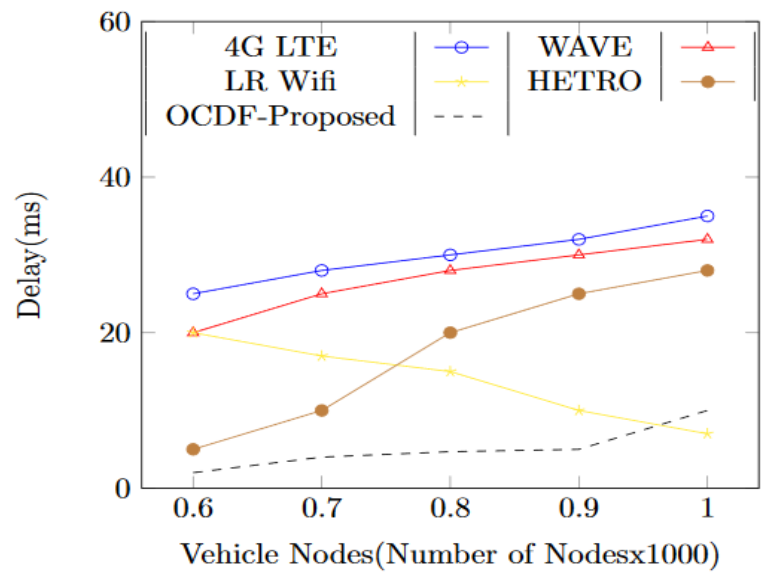

Fig. 4. Vehicle nodes vs Delay [9]

\section{Discussions}

In our work, we have considered 4G LTE, LR Wi-Fi, WAVE, HETRO techniques to compare with our proposed algorithm to evaluate the performance of throughput and delay in IoV environment. Figure 3 shows the throughput performance that has been increased nearly 50\% higher than the 4G LTE, 40\% than WAVE, 30\% than LR Wi-Fi, 25\% higher than HETRO which shows superior than the existing algorithms. The higher throughput is achieved due to less packet drops with the proposed tech- 
niques. However, results showed that there is a slight decline as the vehicle density and speed increases. This degradation is seen due to couple of reasons such as unnecessary handovers, handover delays, and inappropriate selection of target networks at higher speeds. In contrast, figure 4 shows the delay performance and its comparison with the existing approaches. Larger the delay will affect the QoS performance of delay-sensitive applications. Lesser delays can be achieved by selecting the networks well before approaching the access points which is the main goal of our proposed work.

\section{Conclusion}

This paper has presented a brief review of the existing heterogeneous architectures to integrate multiple radio access technologies in IoV. Further, techniques associated with integrated multiple RAT is deeply studied and a detailed comparison is done highlighting the gaps and inconsistencies. In our work, we have computed throughput and delay to demonstrate the performance of proposed algorithm. Moreover, a detailed comparison is done with the existing algorithms using NS-3 simulation tool. The paper has conducted simulations assuming urban road scenarios as the existing works had shown lack of investigations under urban scenarios. It is critically important to evaluate the performance under high vehicle density scenarios especially when it comes to safety-critical applications where the delay and throughput parameters are really challenging. Developing algorithms to enhance the performance of vehicular applications is still a major requirement without sacrificing the QoS requirements. In our work, we have shown superiority of our algorithm in terms of throughput and delay in comparison to the state-of-the-art. Our future work is mainly focused on reducing the handover delays when selecting the radio access technology as increased delays may lead to packet losses.

\section{Acknowledgement}

I would like to thank my supervisor for his continuous support and guidance throughout my research work and also, my sincere thanks to Universiti Teknologi Malaysia (UTM), Johor Bahru for facilitating with all the resources that are required for my research study.

\section{$9 \quad$ References}

[1] L. A. Maglaras, A. H. Al-Bayatti, Ying He, I. Wagner and H. Janicke, "Social Internet of Vehicles for Smart Cities," Sensors and Actuators Networks, vol. 5, no. 1, pp. 1-22, 2016. https://doi.org/10.3390/jsan5010003

[2] K. Zheng, Q. Zheng, P. Chatzimisios, W. Xiang and Y. Zhou, "Heterogeneous Vehicular Networking: A Survey on Architecture, Challenges, and Solutions," in IEEE Communications Surveys \& Tutorials, 2015. https://doi.org/10.1109/comst.2015.2440103 
Paper-Integration of 4G LTE and DSRC (IEEE 802.11p) for Enhancing Vehicular Network...

[3] V. K. Gugulothu, C. M. K. Swain and S. Das, "Throughput performance analysis of an integrated mobile WiMAX - DSRC cellular network with adaptive modulation and coding technique," in 2017 2nd IEEE International Conference on Recent Trends in Electronics, Information \& Communication Technology (RTEICT), Bangalore, India, 2017. https://doi.org/10.1109/rteict.2017.8256774

[4] Z. Xu, X. Li, X. Zhao and M. H. Zhang, "DSRC versus 4G-LTE for Connected Vehicle Applications: A Study on Field Experiments of Vehicular Communication Performance," Journal of advanced transportation, pp. 1-12, 2017. https://doi.org/10.1155/2017/2750452

[5] N. Soni, R. Malekian, D. Andriukaitis and D. Navikas, "Internet of Vehicles Based Approach for Road Safety Applications Using Sensor Technologies," Wireless Personal Communications, p. \{ \}, 2019. https://doi.org/10.1007/s11277-019-06144-0

[6] A. Thakur and R. Malekian, "Internet of Vehicles Communication Technologies for Traffic Management and Road Safety Applications," Wireless Personal Communications volume, vol. 109, pp. 31-49, 2019. https://doi.org/10.1007/s11277-019-06548-y

[7] S. U. Bhover, A. Tugashetti and P. Rashinkar, "V2X communication protocol in VANET for co-operative intelligent transportation system," in 2017 International Conference on Innovative Mechanisms for Industry Applications (ICIMIA), Bangalore, India, 2017. https://doi.org/10.1109/icimia.2017.7975531

[8] F. B. N. U. I. Kashif Naseer Qureshi, "Link Aware High Data Transmission Approach for Internet of Vehicles," in 2019 2nd International Conference on Computer Applications \& Information Security (ICCAIS), Riyadh, Saudi Arabia, Saudi Arabia, 2019. https://doi.org/10.1109/cais.2019.8769482

[9] H. H. R. Sherazi, Z. A. Khan, R. Iqbal, S. Rizwan, M. A. Imran and K. Awan, "A Heterogeneous IoV Architecture for Data Forwarding in Vehicle to Infrastructure Communication," Mobile Information Systems, vol. 2019, p. 12, 2019. https://doi.org/10.1155/ $\underline{2019 / 3101276}$

[10] S. Z. \&. J. A. G. I. Juan Contreras-Castillo, "A seven-layered model architecture for Internet of Vehicles," Journal of Information and Telecommunication, pp. 1-22, 2017.

[11] S. Ansari, T. Boutaleb, S. Sinanovic, C. Gamio and I. Krikidis, "On the design and deployment of multitier heterogeneous and adaptive vehicular networks," in 11th International Symposium on Communication Systems, Networks, and Digital Signal Processing (CSNDSP 2018, Glascow, 2018. https://doi.org/10.1109/csndsp.2018.8471807

[12] M. Priyan and G. U. Devi, "A survey on internet of vehicles: applications, technologies, challenges and opportunities," International Journal of Advanced Intelligence Paradigms, vol. 12, no. 98, pp. 98 - 119, 2019. https://doi.org/10.1504/ijaip.2019.10017745

[13] D. S. f. E. F. C. D. C. i. H. L.-D. V. Networks, "Duplicate suppression for efficient floating car data collection in heterogeneous LTE-DSRC vehicular networks," Computer Communications, vol. 123, pp. 54-64, 2018. https://doi.org/10.1016/j.comcom.2018.03.015

[14] M. M. M. Nasr, A. M. S. Abdelgader, Z.-G. Wang and L.-F. Shen, "VANET Clustering Based Routing Protocol Suitable for Deserts," Sensors, vol. 16, pp. 1-23, 2016. https://doi.org/10.3390/s16040478

[15] S. Ucar, S. C. Ergen and O. Ozkasap, "Multihop-Cluster-Based IEEE 802.11p and LTE Hybrid Architecture for VANET Safety Message Dissemination," IEEE Transactions on Vehicular Technology, pp. 2621 - 2636, 2016. https://doi.org/10.1109/tvt.2015.2421277 


\section{Authors}

Shaik Mazhar Hussain, Department of Communications Engineering, Advanced Telecommunication Technology (ATT) received his Master's degree in Embedded System, JNTU, Hyderabad, India, PGCert from Coventry University, UK. He is currently a Ph.D. candidate at the Universiti Teknology Malaysia (UTM), Johor Bahru, Malaysia. His research areas of interests include Internet of Things (IoT) and Intelligent Transportation System (ITS), Artificial Intelligence, Wireless Communications. Email: hussain@graduate.utm.my

Kamaludin Mohamad Yusof, School of Electrical Engineering, Universiti Teknologi Malaysia (UTM), Skudai Johor. Graduated with a Bachelor of Electrical Engineering, Master of Electrical Engineering from UTM and Ph.D. in Electrical engineering, University of Essex, UK. He has involved in several research areas such as Internet of Thing, Internet Networking and Network Security. Email: kamalmy@utm.my, kamalmy@fke.utm.my

Shaik Ashfaq Hussain, Department of Communications Engineering, Advanced Telecommunication Technology (ATT) received his Master's degree in Embedded and VLSI System design from India in 2013. He is currently a Ph.D. candidate at the Universiti Teknology Malaysia (UTM), Johor Bahru, Malaysia. His research areas of interests include Internet of Things (IoT) and Intelligent Transportation System (ITS), Artificial Intelligence, Wireless Communications. Email: sk.ashfaq.hussain@icloud. com

Mr. Rolito Asuncion, Department of Electronics and Communications Engineering, Middle East College, Oman. Graduated with a Bachelor of Electronics and Communication (1990) from University of Santo Tornas and Master of Electrical Engineering (2000) from Mapua Institute of Technology. His research areas of interest include Linear Control Engineering, Robotics, and PLC-SCADA. Email : rolito@mec.edu.om

Syed Ghouse, Department of Information Technology, received his Bachelor's degree in Information Technology (IT) from JNTU, Hyderbad, India. And also received his Master's degree in Information Technology (IT) from JNTU, Hyderbad, India. His research areas of interests include Wireless mobile communication systems, Network routing protocols (RIP, IGP, EIGRP, OSPF \& BGP), Intelligent Transportation System (ITS) and Internet of Things (IoT). Email : isyedghouse@ gmail.com

Article submitted 2020-10-13. Resubmitted 2021-05-24. Final acceptance 2021-05-26. Final version published as submitted by the authors. 